\title{
Ion Beam Therapy
}

\section{Sabin JR* \\ Department of Fysik, Kemi, ogFarmaci, University of Southern Denmark, USA}

*Corresponding author: Sabin JR, Quantum Theory Project, Department of Physics, University of Florida, USA, and Department of Fysik, Kemi, ogFarmaci, University of Southern Denmark, Odense, Denmark, E-mail: sabin@qtp.ufl.edu

\section{Editorial \\ Volume 1 Issue 1}

Received Date: November 16, 2017

Published Date: December 15, 2017
Of the various treatments for cancer, one of the most used over recent years has been radiation. Originally, the radiation used was x-rays as its properties, both physical and medical, have been well known for many years. The treatment is due to deposition of $x$-ray beam energy into the molecules in the tumor, destroying them and thus killing the tumor. In Figure 1, the radiation dose from an x-ray beam is plotted as a function of depth in tissue. The position of the peak depends on the $\mathrm{x}$-ray energy, i.e. the frequency. It is clear, however, that energy is not deposited at a single depth, but continuously along the pathway, and thus healthy tissue surrounding the tumor will also be damaged. Similar arguments apply to all photon beams.

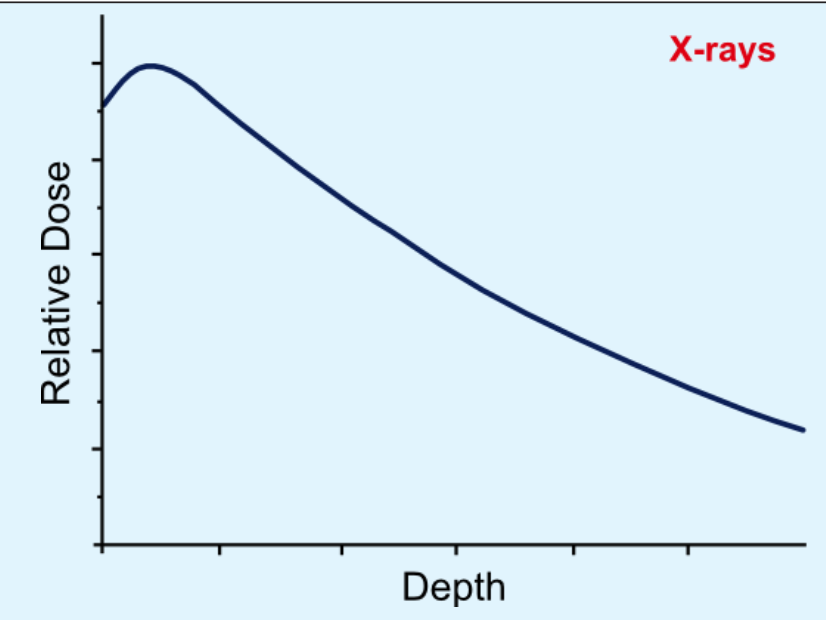

Figure 1: Energy deposited vs. depth of X-rays penetrating tissue.

More recently, baryon beams, most frequently protons, have been used for tumor treatment, again by depositing beam energy into molecules in a tumor. As in Figure $1 \& 2$ presents the radiation dose plotted as a function of depth in tissue, but this time for a proton beam. As in the case of $x$-rays, the location of the peak, known as the Bragg peak, depends on the energy of the beam, that is, the velocity of the protons. As can be seen, when the proton velocity is selected so that the Bragg peak is located on the tumor, there is much less damage to surrounding tissue that for an $\mathrm{x}$-ray beam.

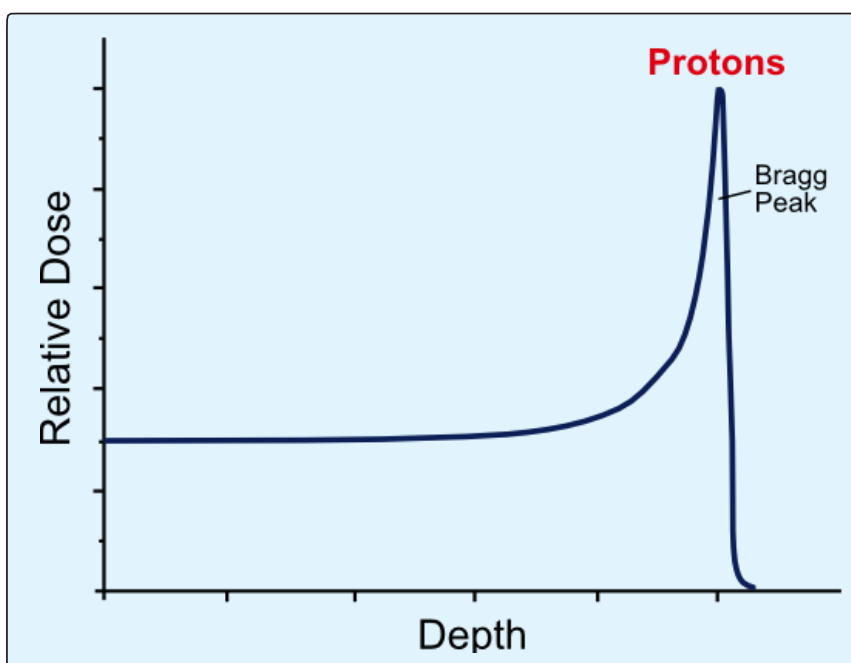

Figure 2: Energy deposited vs. depth of swift protons penetrating tissue.

As tumor therapy depends on destruction of molecules in the tumor, the next question to be considered concerns how much beam energy can be transferred to a particular molecule in the tumor, such energy transfer will be a sum of energy transfer from a single beam particle to a molecule. Knowing the target system's density (n) and particle velocity (v), the beam particle energy loss can be determined:

$$
-\frac{d E}{d x}=n S(v)
$$

where $\mathrm{S}(\mathrm{v})$ is the stopping cross section of the target. If the target is an atom, knowing the identity of and electronic excitation characteristics of the target allows direct calculation of the stopping cross section $[1,2]$ of an atom from: 


$$
-\frac{d E}{d x}=n S(v)
$$

Here $\mathrm{Z} 1$ and $\mathrm{Z} 2$ are the projectile charge and target electron number, respectively, and I0is the mean excitation energy of the target which indicated the ability of the target to absorb energy from the projectile, and is calculated from the target dipole oscillator strength distribution (f):

$$
\ln I_{0}=\frac{\int \frac{d f}{d E} \ln E d E}{\int \frac{d f}{d E} d E}
$$

In a tumor situation, however, the radiation target is not an atom, but a biological molecule. Thus it is necessary to invoke the Bragg Rule [3], which states that the stopping cross section of an aggregate of fragments, such as a molecule or a functional group, can be well approximated by a sum of the stopping cross sections of the fragments:

$$
S_{\text {aggrigate }}(v)=\sum_{i=\text { fragments }} S_{i}(v)(4)
$$

When expressed in terms of fragment mean excitation energies, this becomes:

$$
S_{\text {aggrigate }}(v)=\frac{1}{N_{e}} \sum_{i=\text { fragments }} \omega_{i} \ln I_{0}^{1}(5)
$$

Here the fragment can be an atom, or some molecular functional group, and $\mathrm{Ne}$ is the number of electrons in the fragment, and is the electron number of fragment $i$. We have calculated such quantities for several such situations [4]. Thus, knowledge of the stopping power of atoms and functional groups allows determination of the stopping power, or energy absorption, of molecules located in tumors, and thus destruction of the tumor [5].

Note: A review of the non-mathematical side of tumor therapy with proton beams is available in: Harald Paganetti, Proton Beam Therapy, IOP Publishing Ltd., 2017. See also [6-9].

\section{References}

1. (1981) More detailed presentations of stopping may be found in Bonderup E,"Penetration of Charged Particles through Matter,"Fysik Institutes trykkeri, Århus Universitet, 2nd (edn), Århus.

2. Bragg WH, Kleeman R (1905) On the Alpha Particles of Radium and Their Loss of Range on Passing through Various Atoms and Molecules. Philos Mag 10(57): 318-340.

3. Sabin JR (2013) "On the Calculation of Biomolecular Mean Excitation Energies". J Phys Chem Biophys 3: $1 \mathrm{e} 110$

4. Sabin JR (2015) "Stopping Power of Biological Systems". J Phys Chem Biophys 5: 2, e125.

5. Jensen PWK, Sauer SPA, Oddershede J, Sabin JR (2017) "Mean Excitation Energies for Molecular Ions” Nucl Instr Meth B 394, 73.

6. cf. e.g. Sauer SPA, Oddershede J, Sabin JR (2011) "Mean Excitation Energies for Biomolecules: Glycine to DNA," Adv. Quantum Chem 62: 215-243.

7. Moribayashi K (2016) "Simulation Study of Radial Dose due to the Irradiation of a Swift Ion Aiming to Advance the Treatment Planning System for Heave Particle Cancer Therapy

8. (2015) The Effect of Emission Angles of Secondary Electrons," Nucl Instur Meth 365, 592

9. Story M, Pompos A, Timmerman R "On the Value of Carbon-Ion Therapy," Phys. Today 69(11): 14. 